lacks vasopermeability-inducing effects, it might not be time to pull the plug on the development of high-dose systemic IL-2 therapies after all. To this effect, Epstein and colleagues have shown that a point mutation at amino-acid 38 of IL-2 - in the 22-58 amino-acid PEP region - completely blocks the vasopermeability effect but does not affect cytokine activity. Conversely, pre-treatment with tumour-targeting antibody-PEP fusion proteins is being developed as a strategy to enhance drug delivery to tumours, such that higher effective doses of chemotherapy can be targeted to tumours without additional systemic side effects.

(2) References and links ORIGINAL RESEARCH PAPERS Epstein, A. L. et al. Identification of a protein fragment of interleukin 2 responsible for vasopermeability. J. Natl Cancer Inst. 95, 741-749 (2003) | Hu, P. et al. The generation of low toxicity interleukin-2 fusion proteins devoid of vasopermeability activity. Blood 101, 4853-4861 (2003)

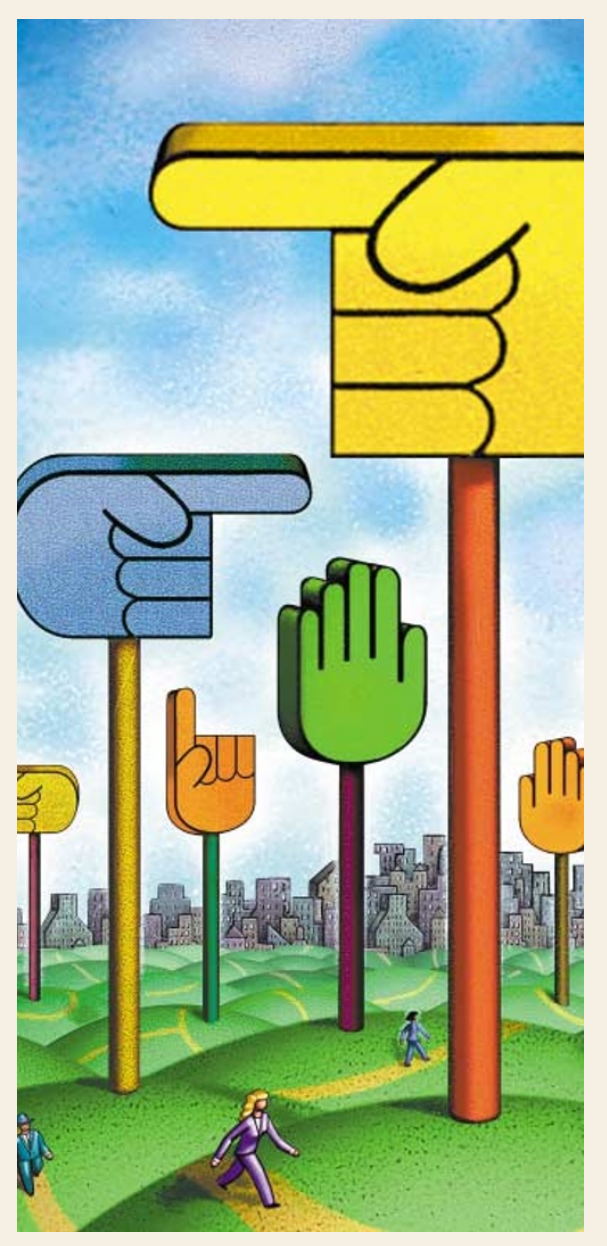

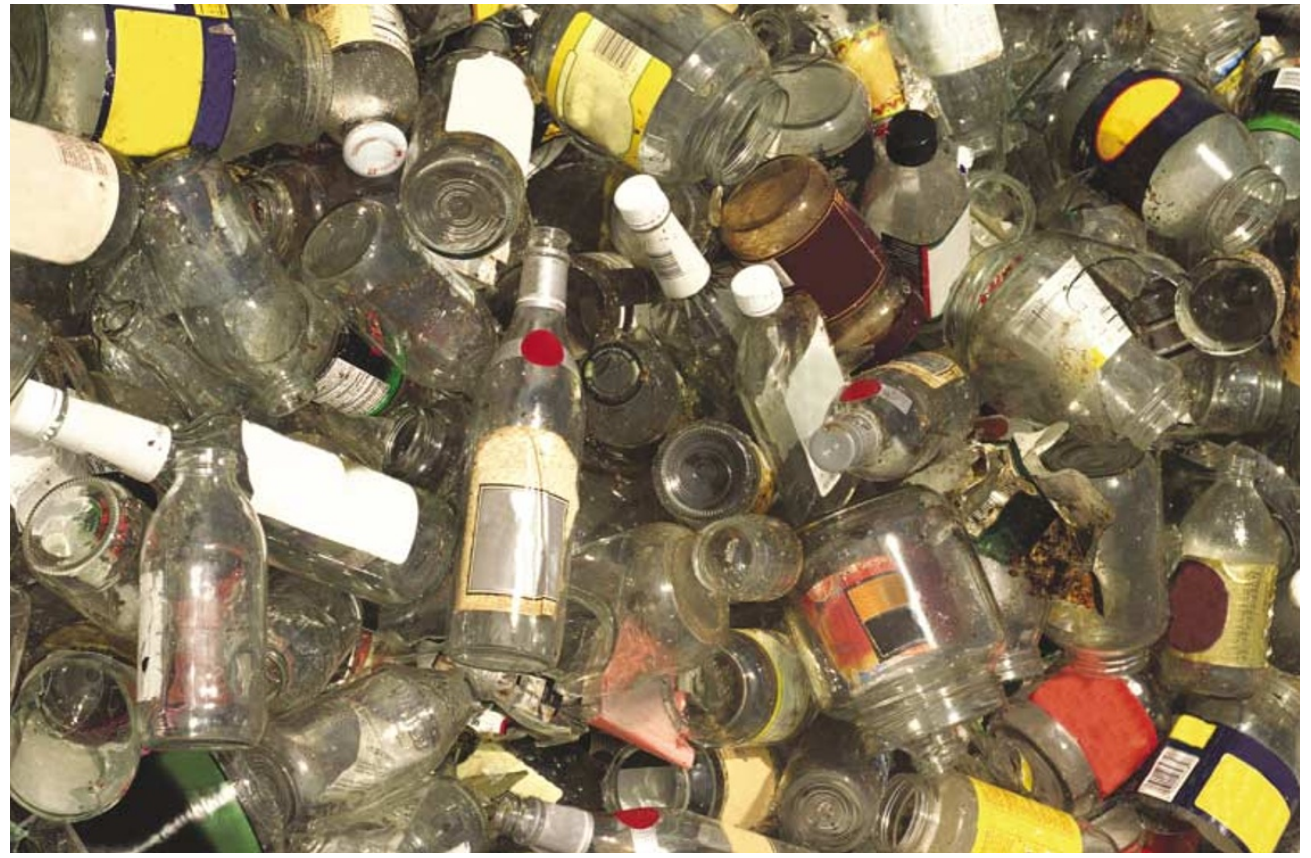

TUMORIGENIC PATHWAYS

\section{Unruly recycling}

Recycling is thought to be good for the environment, but this might not be true if you are a cancer cell. Endocytic pathways that recycle growth-factor receptors from the surface of the cell limit the activation of tumorigenic signalling pathways, so altering receptor trafficking could be a mechanism of inducing transformation. Now, Theodora Ross and colleagues report the first example of how cancer cells manipulate endocytosis to their own end.

Huntingtin interacting protein-1 (HIP1) is a cofactor in clathrin-mediated endocytosis that has been found to be upregulated in various human epithelial cancers. To investigate whether it could have a causal role in inducing transformation, the authors generated fibroblasts that stably expressed HIP1. HIP1 overexpression was found to confer on cells the ability to grow in the absence of anchorage, to form foci when plated at low density and to form tumours when injected into immunodeficient mice, all of which are markers of transformation. HIP1-expressing cells had a higher rate of proliferation than control cells and, interestingly, were able to proliferate in only $0.1 \%$ serum - under which conditions RAStransformed cells were unable to grow indicative of an increased sensitivity to growth factors.

So, how might HIP1 expression affect the growth-factor signalling pathway? Ross and colleagues first investigated the epidermal growth-factor receptor (EGFR) pathway, and found that EGFR was upregulated and surface levels of EGFR were increased. EGFR was also found to be activated - demonstrated by its tyrosine phosphorylation - as were several downstream components, including the ERK cascade and PI3K, but not PLC $\gamma$.
Despite these observations, the question of how HIP1-expressing cells grow in low serum had not been answered. The idea that the cells might secrete growth factors was ruled out, as it was found that conditioned media from HIP1expressing cells was unable to support growth in low serum of the EGF-sensitive cell line MCF10A. EGFR activity was also not constitutive, as the presence of EGF in the media could stimulate proliferation, and an EGFR inhibitor could reduce proliferation. However, not all aspects of the altered growth and transformation were inhibited by the EGFR inhibitor, which indicates that other growth-factor pathways are involved.

Further analysis confirmed this, as the fibroblast growth-factor receptors 3 and 4 were upregulated in HIP1-expressing cells. The mechanism by which HIP1 might affect several growth-factor receptors was hypothesized to be through clathrin trafficking, as HIP1 was known to interact with clathrin and the endocytic adaptor protein AP2. Indeed, in HIP1-expressing cells, AP2 is downregulated and clathrin was more concentrated at the Golgi network, both of which could result in a decrease in receptor uptake, recycling and degradation.

So, HIP1 transforms cells by altering endocytosis, resulting in growth-factor-receptor accumulation on the cell surface. But is this actually relevant in human cancer? By analysing the expression of HIP1 and EGFR in multiple, primary breast cancers, the authors showed that HIP1 expression tended to increase as breast tissue progressed from normal to invasive carcinomas, and that EGFR expression correlated with that of HIP1. HIP1 might therefore make a useful prognostic marker and therapeutic target.

Emma Greenwood

(2) References and links

ORIGINAL RESEARCH PAPER Rao, D. S. et al. Altered receptor trafficking in Huntingtin interacting protein-1 transformed cells. Cancer Cell 3, 471-482 (2003)

WEB SITE

Theodora Ross's lab:

http://www.med.umich.edu/cmb/faculty/rossts.htm 